\title{
Calbindin Influences Response to Photic Input in Suprachiasmatic Nucleus
}

\author{
Toshiyuki Hamada, ${ }^{1}$ Joseph LeSauter, ${ }^{2}$ Maria Lokshin, ${ }^{1}$ Maria-Teresa Romero, ${ }^{3}$ Lily Yan, ${ }^{1}$ Judith $M$. Venuti, ${ }^{4}$ and \\ Rae Silver ${ }^{1,2,5}$ \\ Departments of Psychology, ${ }^{1}$ Columbia University and ${ }^{2}$ Barnard College, New York, New York 10027, ${ }^{3}$ Department of Psychology, Binghamton University, \\ State University of New York, New York 13902, ${ }^{4}$ Department of Cell Biology and Anatomy, Louisiana State University Medical Center, New Orleans, \\ Louisiana 70112, and ${ }^{5}$ Department of Anatomy and Cell Biology, Columbia University, New York, New York 10027
}

\begin{abstract}
It is well known that light resets the circadian clock only at specific times of day. The mechanisms mediating such gating of environmental input to the CNS are not well understood. We show that calbindin ${ }_{\mathrm{D} 28 \mathrm{~K}}(\mathrm{CalB})$-containing cells of the suprachiasmatic nucleus (SCN), which are directly retinorecipient, gate photic entrainment of cellular circadian oscillators and thereby determine the timing of locomotor rhythmicity. Specifically, we demonstrate a circadian rhythm of subcellular localization of CalB: whereas the protein is detected at all times in the cytoplasm, it is low or absent in the nucleus during the night. Under normal circumstances, light-induced behavioral phase shifts and Period (Per) gene expression in the SCN occur only during the subjective night. Surprisingly, both behavioral phase shifts and light-induced Per are blocked during the subjective night and enhanced during the subjective day after administration of CalB antisense oligodeoxynucleotides. These results suggest a cellular basis for temporal gating of photic input to the circadian clock.
\end{abstract}

Key words: suprachiasmatic nucleus; calbindin ${ }_{\mathrm{D} 28 \mathrm{~K}}$; Per1; vasopressin; digoxigenin in situ hybridization; locomotor activity rhythm

\section{Introduction}

Virtually all organisms synchronize their daily activities to environmental light: dark cycles. In mammals, the circadian clock that entrains the organism to its environment is located in the suprachiasmatic nuclei (SCNs) of the hypothalamus (for review, see Moore, 1996). It is well established that, in both nocturnal and diurnal mammals, light during the night resets or phase shifts the clock, whereas light exposure during the day does not. The daily resetting of the body clock is essential for correct phasing of internal and external responses necessary for survival, including sleep-wake cycles, coordination of hormone rhythms, metabolic activity of cells, etc. These phase-dependent responses to light are observed in SCN rhythms of gene expression, electrical activity, and behavioral and physiological responses (Klein et al., 1991; King and Takahashi, 2000).

We reported previously that the hamster SCN is marked by a densely packed population of calbindin (CalB)-containing cells in its caudal aspect [termed the "CalB region" (Silver et al., 1996; Hamada et al., 2001) or "SCN core" (Moore, 1996)]. These cells receive direct retinal input, revealed by double-label electron mi-

\section{Received June 9, 2003; revised Aug. 1, 2003; accepted Aug. 6, 2003.}

This research was supported by National Institutes of Health Grant NS37919 (R.S.) and the Japan Society for the Promotion of Science (T.H.). We thank Dr. M. C. Antle for assistance in all aspects of manuscript preparation. We also thank Drs. A. Bult, P. Hardin, and W. J. Schwartz for helpful comments on previous drafts of this manuscript and H. Kirwan for technical assistance.

Correspondence should be addressed to Rae Silver, Columbia University, Mail Code 5501, 1190 Amsterdam Avenue, New York, NY 10027. E-mail: qr@columbia.edu.

T. Hamada's present address: Age Dimension Research Center, National Institute of Advanced Industrial Science and Technology, Advanced Industrial Science and Technology Tsukuba Central Sixth Site 1-1-1, Higashi, Tsukuba, Ibaraki, 305-8562, Japan.

Copyright $\odot 2003$ Society for Neuroscience $\quad 0270-6474 / 03 / 238820-07 \$ 15.00 / 0$ croscopy (EM) (Bryant et al., 2000), and they express Fos in response to a light pulse (Silver et al., 1996). Furthermore, constitutively high expression of Period 3 (Per3) mRNA and phasedependent light-induced expression of both Per1 and Per2 mRNA are concentrated in the CalB subnucleus. In comparison, rhythmic Per 1, Per 2 , and Per $3 \mathrm{mRNA}$ occur in a region marked by vasopressin (VP)-containing neurons [termed the "VP region" (Hamada et al., 2001) or "SCN shell" (Moore, 1996)]. These results form the rationale for the present studies on the role of CalB in light-induced phase shifts of locomotor rhythms and Per mRNA expression. To understand its role in the SCN, we first examined the circadian properties of CalB-containing cells. Next, we decreased CalB protein levels using antisense oligodeoxynucleotides (AS-ODNs) and examined effects on behavioral phase shifts and SCN gene expression in response to photic stimuli. As has been noted, the use of antisense technology as a tool in neuroscientific research permits the reversible knock-down of gene expression, with significant temporal and spatial specificity (Ogawa and Pfaff, 1998; Estibeiro and Godfray, 2001).

\section{Materials and Methods}

Animals and housing. Adult male hamsters (Mesocricetus auratus) from Charles River Laboratories (Wilmington, MA) were maintained in $12 \mathrm{hr}$ light/dark cycle $(\mathrm{LD})$ in polypropylene cages $(20 \times 40 \times 20 \mathrm{~cm})$. Animals had ad libitum access to food and water. The room was maintained at $23 \pm 1^{\circ} \mathrm{C}$. Hamsters were kept in LD for at least 2 weeks before being tested. Hamsters tested in constant darkness (DD) were transferred to a dark room for 1 week and were housed in cages equipped with running wheels (diameter, $16 \mathrm{~cm}$ ). A dim red light ( $<1$ lux; Delta 1, Dallas, TX) allowed for animal maintenance, and a white-noise generator $(91 \mathrm{~dB}$ sound pressure level) masked environmental noise. Locomotor activity was monitored continuously using a computer-based data acquisition 
system (Dataquest; Data Sciences, St. Paul, MN). Actograms were printed, and a regression line fit to daily onsets was used to predict activity onset on the day of manipulation. Manipulations were timed relative to predicted activity onset, which is conventionally defined as circadian time (CT) 12. Phase shifts were determined in behavioral studies by fitting a second regression line to activity onsets starting $3 \mathrm{~d}$ after treatment. The horizontal distance between the two regression lines on the day after treatment represented the change in phase.

Surgery and perfusion. A stainless steel guide cannula (Plastics One, Roanoke, VA) was stereotaxically implanted into the third ventricle (3V) using the following coordinates: $1.0 \mathrm{~mm}$ anterior, $0.0 \mathrm{~mm}$ lateral to bregma at depth of $7.6 \mathrm{~mm}$ below the skull. After surgery, hamsters were housed in DD and allowed to establish stable free-running activity rhythms. After experimental manipulations, animals were deeply anesthetized and perfused intracardially with $4 \%$ paraformaldehyde in $0.1 \mathrm{M}$ phosphate buffer. Brains were harvested, postfixed in $4 \%$ paraformaldehyde overnight, and then cryoprotected in $20 \%$ sucrose for $24 \mathrm{hr}$. Brains were then frozen with dry ice and were stored at $-80^{\circ} \mathrm{C}$.

Injection materials. The gel-purified phosphothionate ODNs (Genelink, Hawthorne, NY) used were CalB-AS (5'-AGGTGCGATTCTGCCATGG-3'), VP-AS (5' -TCATGGCGAGCATAGGTGG-3'), scrambled (S)-AS ( $5^{\prime}$-ATCTAGGCGTCGCTCATAC-3'), and CalB-S (5'-CCATGGCAGAATCGCACCT- $\left.3^{\prime}\right)$. These were dissolved in isotonic sterile saline. Some animals received an intraperitoneal injection of 8-hydroxy-2(dipropylamino)tetralin (8-OH DPAT) ( $5 \mathrm{mg} / \mathrm{kg}$; Sigma, St. Louis, MO), also dissolved in isotonic sterile saline.

Experiment 1: circadian properties of CalB. Brains from hamsters killed at various times in either LD or DD were processed for CalB immunoreactivity (IR). Serial sections through the hypothalamus were collected for light (10 $\mu \mathrm{m}$ sections), immunofluoresence (40 $\mu \mathrm{m}$ sections), and confocal (50 $\mu \mathrm{m}$ sections) microscopy and were processed with immunocytochemistry, as described by Silver et al. (1996). Briefly, the tissue was incubated in a monoclonal mouse CalB antibody (Sigma) at a concentration between 1:20,000 (fluorescence microscopy) and 1:40,000 [light microscopy (LM)] for $48 \mathrm{hr}$. After this, the location of the primary antibody was visualized using one of three methods: immunofluorescence (FITC), immunoperoxidase staining (SG chromogen; Vector Laboratories, Burlingame, CA) for conventional microscopy, or immunofluorescence (Cy2) for confocal microscopy. The brains from other hamsters were processed for immunoelectron microscopy as described by Bryant et al. (2000).

To quantitate the intracellular localization of CalB protein in the SCN, the proportion of cells with nuclei devoid of CalB immunoreactivity were counted by two independent investigators blind to the experimental conditions. For FITC-stained material, sections from animals killed at two time points (CT6, $n=2$; CT22, $n=4 ; 150-550$ cells per animal) were observed under a fluorescent microscope (Nikon E800; Nikon, Tokyo, Japan). Images were captured with a cooled CCD camera (SPOT; Morrell, Melville, NY). These images were used to count the total number of cells and the cells with empty nuclei. The percentage of cells with empty nuclei was compared between groups. For confocal microscopy a Zeiss (Tornwood, NY) Axiovert 100 TV with a laser scanning confocal attachment was used (Zeiss LSM 410). The sections were excited with an argonkrypton laser using the wavelength for FITC (488 nm), and images were collected as $1-\mu \mathrm{m}$-thick optical sections. Images were processed using the Zeiss LSM program (version 3.92). The proportion of cells with nuclei devoid of CalB-IR was counted as above $(n=150-250$ cells per animal; CT6, $n=2$; CT22, $n=4$ ). For electron microscopy, photographs from randomly chosen ultrathin sections were examined, and the proportion of cells with nuclei devoid of CalB-IR was determined (CT6, $n=$ 2 ; CT22, $n=2$ ). Animals in these three groups were combined for statistical analysis ( $t$ test), although the data are shown for each group. For the group tested under LD, sections stained with SG were observed under light microscopy as above (CT6, $n=4$; CT20, $n=7$ ). For the final group, 22 animals were kept in DD for 1 week and were killed as follows: CT0CT4, CT4-CT8, CT8-CT12, CT12-CT16, CT16-CT20, and CT20CT24. Sections are $10 \mu \mathrm{m}$ were labeled with SG chromogen and observed under a light microscope (Olympus BH-2; Olympus Optical, Tokyo, Japan). The average diameter of the SCN CalB cells was $10.7 \pm 0.18 \mu \mathrm{m}$, and that of the nuclei was $9.17 \pm 0.06 \mu \mathrm{m}$, measured using NIH Image in 40 cells with nuclei devoid of CalB-IR. The relative optical density (ROD) of the nuclei and cytoplasm was measured in 5-35 cells for each animal. In cells with nuclei devoid of CalB-IR, measurements of the OD for both the nuclear and the cytoplasmic were performed. In cases when CalB-IR was observed throughout the cell, only the largest cells $(10-12 \mu \mathrm{m})$ were measured to ensure that only cells cut through the nucleus were measured. ROD was determined as follows: a $9-\mu \mathrm{m}$-diameter circle was placed in the middle of the cell, and the OD was measured. Then, the perimeter of the cell was traced, and the OD of the cytoplasm (OD between the two lines) was measured. Finally, the ROD of the cytoplasm and of the nucleus was measured relative to the staining of a nonimmunoreactive region in the SCN outside the CalB subnucleus. The mean ROD for the nucleus and the cytoplasm was calculated for each animal. Cytoplasmic ROD was subtracted from nuclear ROD and analyzed by time of day (see Fig. 1C).

For CalB protein quantification, sections were observed under confocal microscopy as above, except that the images collected were $2-\mu \mathrm{m}$ thick optical sections. The ROD of CalB-IR was measured by subtracting the OD of background from the OD of overall CalB-IR in the subnucleus. The proportion of cells with nuclei devoid of CalB-IR was also counted.

To compare the relationship of CalB localization with behavioral phase shift resulting from light exposure, a phase response curve was determined. Hamsters were given a light pulse (600 lux, $30 \mathrm{~min})$ at the following times of day: CT0 $(n=4)$, CT4 $(n=3)$, CT8 $(n=4)$, CT12 $(n=5)$, CT13.5 $(n=3)$, CT14.5 $(n=4)$, CT15.5 $(n=4)$, CT16 $(n=6)$, CT17.5 $(n=3), \operatorname{CT} 19(n=5)$, CT21 $(n=2)$, and CT22 $(n=3)$. Phase shifts were determined as described above.

Experiment 2: effects of AS-ODN treatment on CalB mRNA and protein. To decrease the level of CalB protein in the cell, we injected CalB-ASODNs. The phosphothionate ODNs used are taken up preferentially and rapidly by neurons in the vicinity of hypothalamic injection sites (Ogawa et al., 1995). Also, phosphothionate ODNs are more resistant to degredation than are unmodified ODNs and are still present $8-16 \mathrm{hr}$ after injection (Ogawa et al., 1995). When combined with dose-response and proper control substances (i.e., sense, scambled AS, vehicle control, and AS for unrelated genes), antisense technology allows for the selective, rapid, spatially specific, and reversible knock-down in the expression of a particular gene. Because the animals are otherwise normal, it is possible to avoid interpretive problems inherent in with transgenic knock-out animals (Ogawa and Pfaff, 1998; Estibeiro and Godfray, 2001).

After at least $5 \mathrm{~d}$ of stable running, hamsters received a microinjection of ODN through the guide cannula using a needle attached by polyethylene tubing to a $50 \mu \mathrm{l}$ Hamilton syringe. A $7.5 \mu \mathrm{l}$ aliquot of ODN or control substances was injected to the floor of the $3 \mathrm{~V}$ at specific circadian times. After each injection, the needle was left in place for $5 \mathrm{~min}$. To confirm the successful application of CalB-AS in the SCN, animals were injected with $5 \mu \mathrm{l}$ of biotinylated CalB-AS and killed $4 \mathrm{hr}$ later. Their brains were then processed to confirm cellular uptake of the ODN.

Initially, animals were given subjective day or subjective night intracerebroventricular injections of saline, CalB-S, S-AS, VP-AS or CalB-AS. Animals were killed $4 \mathrm{hr}$ later, and their brains were collected and processed for either CalB-IR or in situ hybridization for CalB mRNA or VP mRNA. In situ hybridization was done using $30 \mu \mathrm{m}$ sections through the SCN as described previously (Hamada et al., 2001). The ROD of Perl, CalB and VP mRNA was quantified using NIH Image as described previously (Hamada et al., 2001).

Experiment 3: effects of AS-ODN on the circadian system. To explore the function of CalB-containing cells, we administered CalB-antisense phosphothioate oligodeoxynucleotides into the $3 \mathrm{~V}$ near the SCN and, $4 \mathrm{hr}$ later, tested the animals for phase shifts during the subjective day and night (for experimental designs, see Figs. $3 A, 4 A, C$ ). Control injections included saline, CalB-S, S-AS, and VP-AS. It has been reported previously that intracerebroventricular injections of AS are taken up by the SCN and that such treatment blocks light-induced phase shifts of locomotor behavior, induction of Per1 and Per 2 mRNA, and electrical rhythms within 1-2.5 hr (Akiyama et al., 1999). To test the effects of light, hamsters were given CalB-AS (or control substances) at CT2-CT4 and, 4 
A
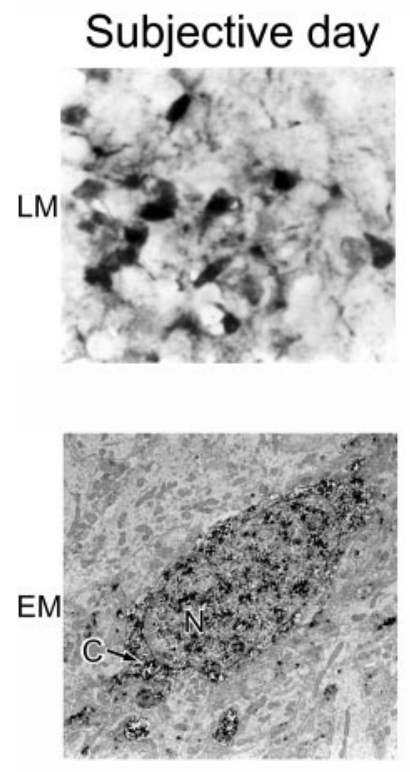

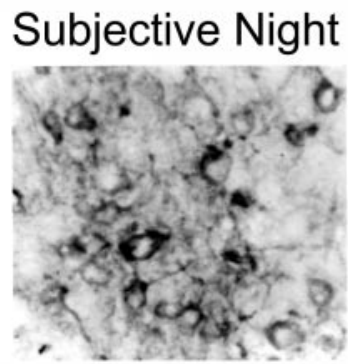

B
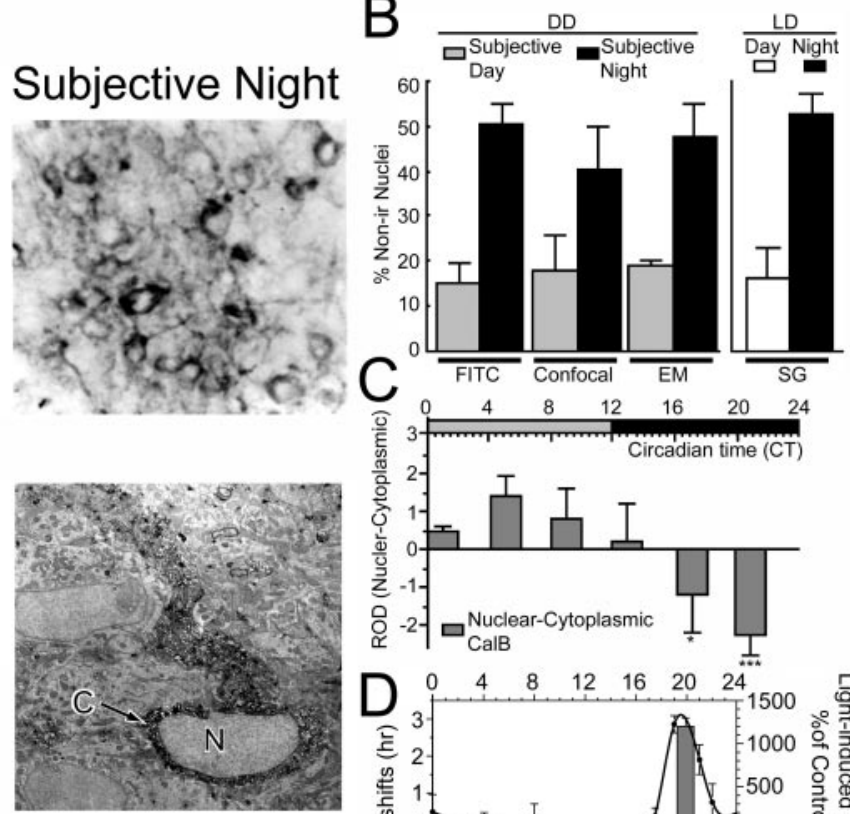

Figure 1. There is a circadian rhythm in nuclear CalB content in the SCN. A, Light microscopic (LM; top panels) and electron microscopic (EM; bottom panels) photomicrographs show the changes in localization of CaIB-IR as a function of time of day with the nuclei are primarily devoid of CalB during the subjective night (CT22), whereas CalB-IR is seen in both cytoplasm and nucleus during the subjective day (CT6). N, Nucleus; C, cytoplasm. B, Percentage of CalB cells with nuclei devoid of CalB-IR using four different methods. Histograms show the mean \pm SEM. C, Histograms show the nuclear versus cytoplasmic ROD of CalB throughout the circadian cycle. Positive values indicate that the staining intensity is greater in the nucleus than in the cytoplasm, and negative values indicate that staining intensity is less in the nucleus than in the cytoplasm. D, Schematic summarizing the temporal relationship between light (600 lux, 30 min)-induced phase shifts of locomotor activity (line graph) and light-induced Per1 mRNA expression in the CalB region (histograms). The phase response curve was determined using a 30 min light pulse (600 lux; $2-5$ animals per time point). Histograms depict data as mean \pm SEM. ${ }^{*} p<0.05 ;{ }^{* * *} p<0.001$.

hr later, were given a light pulse (600 lux, $30 \mathrm{~min})$. To assess whether this treatment specifically affected only photic phase shifts, some animals were injected with $8-\mathrm{OH}$ DPAT, a treatment that produces nonphotic phase shifts. For anatomical (Per1 mRNA) studies, animals were killed 90 min later (5.5 $\mathrm{hr}$ after injection), and the brains were processed as described above. Animals in behavioral studies were monitored for phase shifts in their locomotor activity as described above.

Statistics. Main effects were examined with ANOVAs, with individual pairwise differences examined using Fisher's PLSD post hoc test. In some cases, Student's $t$ tests were used to examine the difference between two treatments.

\section{Results}

\section{Experiment 1: circadian properties of CalB}

Photomicrographs of LM (top panel) and EM (bottom panel) are presented in Figure $1 \mathrm{~A}$. CalB protein is found in both the nucleus and cytoplasm during the subjective day but is primarily absent from the nucleus during the subjective night. The quantitative analyses are presented in Figure 1, $B$ and $C$, for each of the methods used (FITC, confocal, and EM for DD; light microscopy with SG for LD and for different times of day in DD and LD; see Materials and Methods). In DD, the percentage of CalB cells devoid of immunoreactivity in the nucleus is greater at CT22 than at CT6 $\left(t_{(14)}=7.7 ; p<0.0001\right)$. This difference is also seen in $\operatorname{LD}\left(t_{(9)}=4.6 ; p<0.001\right)$. For quantitative analysis of the localization of the CalB protein as a function of time of day, we examined $10 \mu \mathrm{m}$ sections and used immunoperoxidase labeling with SG as a substrate, because this optimized visualization of the nucleus and cytoplasm. Figure $1 C$ shows significant circadian changes in ROD (see Materials and Methods) in nuclear versus cytoplasmic staining intensity $\left(F_{(5,21)}=3.580 ; p=0.017\right)$ with a peak of nuclear-IR at CT4-CT8 and a trough at CT20-CT24 (CT4-CT8 vs CT16-CT20, $p=0.02$; CT4-CT8 vs CT20-CT24, $p=0.009$; CT8-CT12 vs CT20-CT24, $p=0.02$; CT12-CT16 vs CT20-CT24, $p=0.03$ ).

Light pulses presented at CT4-CT8, the phase of peak nuclear CalB-IR, do not produce phase shifts in locomotor rhythms (Fig. $1 D$, line graph), nor do they induce Per1 expression in the SCN (Fig. $1 D$, bar graph). In contrast, light pulses presented at CT20, corresponding to the trough of nuclear CalB-IR, do induce large phase shifts in locomotor rhythms (Fig. $1 D$, line graph) and significant expression of Per1 in the SCN $\left(F_{(2,10)}=65.773 ; p<\right.$ 0.0001) (Fig. 1D).

\section{Experiment 2: effects of AS-ODN treatment on CalB mRNA and protein}

CalB-AS treatment reduced levels of CalB mRNA in the SCN by $38 \%\left(t_{(4)}=2.84 ; p<0.05\right)$ but had little effect on VP mRNA expression (decrease by $5 \% ; t_{(4)}=0.39 ; p=0.716$ ) (see Fig. $3 A$ ). Similarly, CalB-AS application (injected at CT2-CT4) decreased the content of CalB protein in the SCN by $28 \%\left(t_{(4)}=3.33 ; p<\right.$ 0.05 ) (Fig. $2 B$, right top panel). Importantly, after CalB-AS injection during subjective day (CT2-CT4), the proportion of cells with $\mathrm{CalB}$ protein in their nuclei was significantly decreased $\left(46 \% ; t_{(4)}=3.16 ; p<0.05\right)$ (Fig. $2 B$, right bottom panel), suggesting that the reduced CalB protein content of these cells is primarily attributable to the changes seen in the nuclei. Control treatments of saline, CalB-S, or S-AS did not affect either SCN 

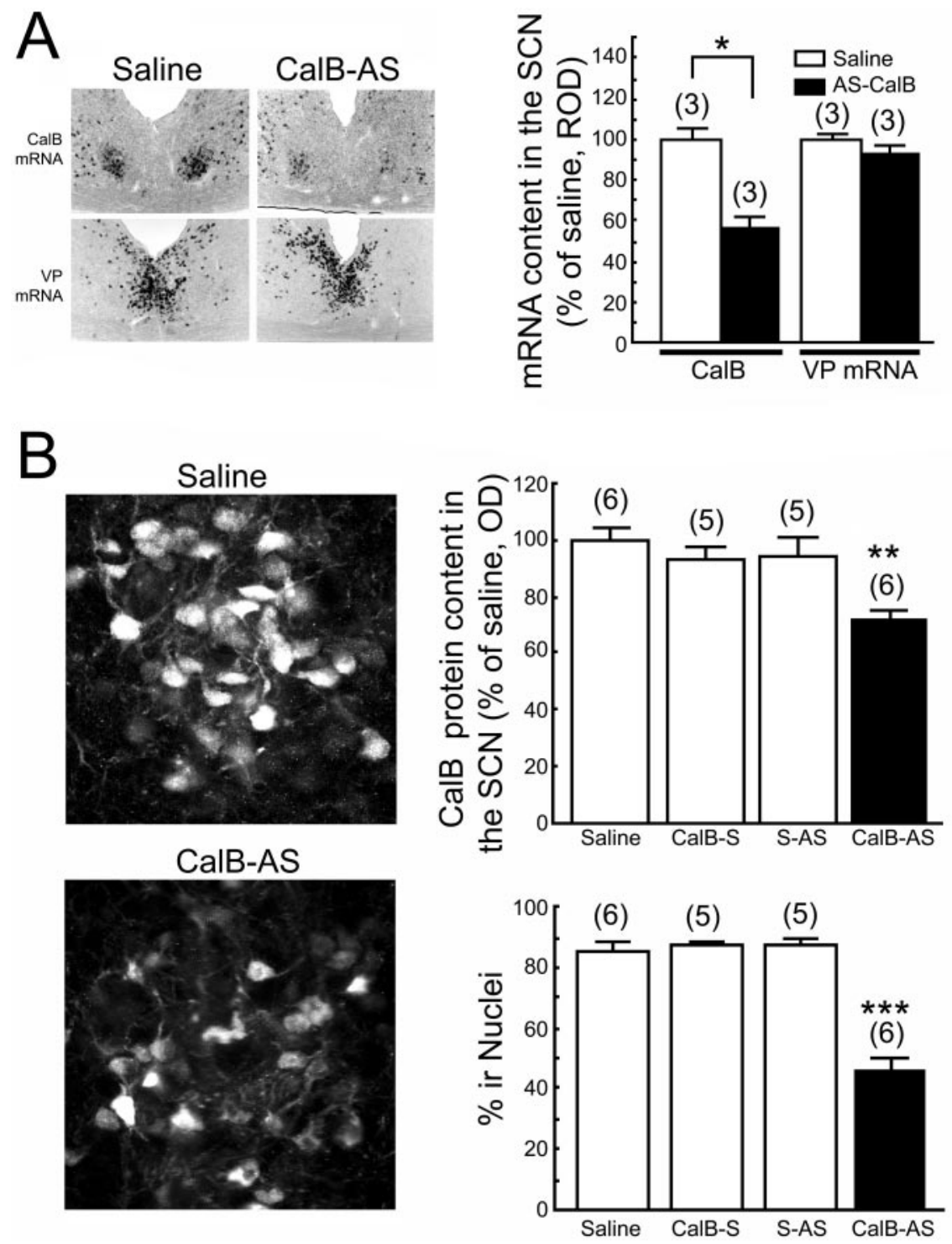

Figure 2. CalB-AS treatment decreases CalB protein and mRNA in the SCN. A, Photomicrographs of CalB and VP mRNA in serial sections demonstrating that $7.5 \mathrm{nmol}$ of $\mathrm{CalB}-\mathrm{AS}$, but not saline, administered at $\mathrm{CT} 4-\mathrm{CT} 6$ significantly reduced the expression of CalB mRNA sampled $4 \mathrm{hr}$ later but did not affect the expression of VP mRNA. B, Confocal images of CalB-IR demonstrating that 7.5 nmol of CaIB-AS, but not saline, administered at CT2-CT4 significantly decreased the OD of Cy2-labeled CaIB protein in the nucleus of SCN cells sampled $4 \mathrm{hr} \mathrm{later.} \mathrm{Histograms} \mathrm{depict} \mathrm{data} \mathrm{as} \mathrm{mean} \pm \mathrm{SEM}$; parentheses give the number of animals per group. * $p<$ $0.05 ;{ }^{* *} p<0.01 ;{ }^{* * *} p<0.001$.

content of CalB protein or percentage of CalB-immunoreactive nuclei (Fig. $2 B$ ).

Experiment 3: effects of AS-ODN on the circadian system During late subjective night (Fig. 3, top panel) CalB-AS blocked light-induced phase advances in a dose-dependent manner $\left(F_{(3,13)}=35.163 ; p<0.0001\right)$ (Fig. 3B, $\left.D-F\right)$.

In contrast, there was no effect of treatment with saline, CalB-S, VP-AS, or S-AS (Fig. 3B-E, G). During subjective night, pretreatment with CalB-AS $4 \mathrm{hr}$ before a light pulse also significantly reduced the expression of light-induced Per1 mRNA $\left(F_{(2,16)}=8.577, p<0.01\right.$; CalB-AS vs saline, $p=0.002$; CalB-AS vs CalB-S, $p=0.017$ ) (Fig. $3 H, I$ ).

During subjective day (Fig. 4, bottom panels) CalB-AS injection followed by a light pulse produced a phase delay of locomotor activity (Fig. $4 B, E$ ), which was significantly different from control animals given saline or CalB-S $\left(F_{(2,10)}=9.818, p<0.01\right.$; CalB-AS vs saline and CalB-S, each $p<0.003$ ) (Fig. $4 E$ ). CalB-AS alone, even at high concentrations $(7.5 \mathrm{nmol})$, had no effect on circadian activity rhythms in the absence of phase-shifting light pulses at any circadian time [mean \pm SEM shift, CT0-CT5, $0.02 \pm 0.01(n=6)$; CT6-CT11, $0.01 \pm$ $0.01(n=6) ;$ CT12-CT17, $-0.01 \pm 0.01$ $(n=4)$; CT18-CT23, $0.00 \pm 0.02(n=$ 4)]. During subjective day, pretreatment with CalB-AS $4 \mathrm{hr}$ before the light pulse resulted in an eightfold increase in lightinduced expression of Per $1 \mathrm{mRNA}$. No increase was seen in any of the control animals $\left(F_{(2,8)}=33.33, p<0.001\right.$; CalB-AS vs saline, $p=0.0004$; CalB-AS vs CalB-S, $p=$ 0.0004) (Fig. 4G,H).

The serotonin $1 \mathrm{~A} / 7$ receptor agonist 8-OH-DPAT produced phase shifts during the subjective day (Fig. $4 D, F$ ). Neither CalB-AS nor control treatments of saline alone or CalB-S had an effect on $8-\mathrm{OH}$ DPAT-induced phase shifts $\left(F_{(2,7)}=\right.$ 0.056; $p=0.95$ ).

As a final control procedure, we examined the effect of the site of AS-ODN administration on the effectiveness of the CalB-AS infusion in blocking lightinduced phase shifts. Precise placement of the cannula at the base of the third ventricle near the SCN was essential for blocking light-induced phase shifts by the CalB-AS. Cannula placements that were positioned unilaterally in the midline region resulted in reduced effectiveness of AS treatment, whereas those that terminated below the optic chiasm were ineffective (data not shown).

\section{Discussion}

It is well documented that light-induced phase shifts of behavior occur only during the night or subjective night. At these times, light also induces the expression of Period genes (Albrecht et al., 1997; Shearman et al., 1997; Shigeyoshi et al., 1997; Reppert and Weaver, 2001). In this paper, we present the following novel findings. (1) There is a correlation between time of day and subcellular localization of CalB protein, with the protein primarily absent from the cell nucleus during the night. (2) Administration of CalB-AS blocks phase shifts and Per1 expression to nighttime light exposure. (3) Impressively, after subjective daytime administration of CalB-AS, the circadian system becomes responsive to light exposure during the subjective day, such that behavioral phase shifts and Per 1 are observed. Together, these results reveal a CalB-based mechanism mediating photic responsiveness of the circadian system.

CalB-containing SCN cells receive direct retinal input (Bryant et al., 2000) but do not express Per1 and Per2 rhythmically (Hamada et al., 2001), and they do not express circadian rhythms in electrical activity (Jobst and Allen, 2002). It is therefore likely 
that CalB cells are important for relaying photic signals to those SCN cells that are not directly retinorecipient. Our results support this view of SCN organization and extend it to specify a role for CalB protein in the transmission of photic information from the ventral SCN to pacemakers in the dorsal SCN. This is consistent with data showing that many CalB cells in the hamster colocalize gastrin-releasing peptide (GRP) (LeSauter et al., 2002), a neuromodulator shown to be important for conveying photic signals from ventral to dorsal SCN in studies of GRP receptordeficient mice (Aida et al., 2002).

Under normal circumstances, light exposure during the subjective day neither phase shifts the circadian clock nor induces Per1 mRNA expression in the SCN. Importantly, our results show that subjective daytime light exposure induces Per1 mRNA expression in the SCN and permits phase delay of locomotor activity when nuclear CalB protein is decreased after CalB-AS treatment. This suggests that CalB protein in the nucleus prevents gene expression in response to photic zeitgebers. Although CalB is important for responses of the circadian system to photic cues, it does not play a role in response to nonphotic stimuli. The serotonin $5-\mathrm{HT}_{1 \mathrm{~A} / 7}$ agonist 8-OH-DPAT is known to produce nonphotic phase shifts (Tominaga et al., 1992), yet these phase shifts are unaffected by CalB-AS treatment. This implies that nonphotic information may be reaching the rhythmic SCN shell through a CalB-independent route.

In performing these studies using antisense technology, we used each recommended control procedure (Nicot and Pfaff, 1997; Branch, 1998; Ogawa and Pfaff, 1998), as follows. The effect of CalB-AS was dose dependent. Nonspecific effects of AS administration was controlled with equivalentvolume saline injections. The scrambled CalB-AS controlled for nucleotide content, demonstrating that the sequence of the AS was the specific cause of the observed effect. CalB-S controlled for any effect of binding to the same region of DNA (the complimentary strand opposite the gene). VP-AS controlled for specificity of CalB-AS, demonstrating that the effect was specific to blocking a particular gene rather than simply attributable to blocking any gene. The fact that equimolar amounts (i.e., $7.5 \mathrm{nmol}$ ) of CalB-S, S-AS, or VP-AS did not result in the same effect as CalB-AS demonstrated that the effect was not attributable to nonspecific effects of administering this dose of oligonucleotides. Furthermore, the desired effect of using CalB-AS (to decrease CalB) was confirmed with both in situ hybridization for CalB mRNA and immunocytochemistry for CalB protein (Fig. 2). The specificity of CalB-AS in affecting CalB and not other genes expressed in the SCN was confirmed by demonstrating no change in VP mRNA after CalB-AS treatment. Finally, the fact that nonphotic phase shifts were not blocked demonstrated that the effect on photic phase shifting was not attributable to general impairment of the functioning of the circadian clock but rather attributable to the impairment of a specific component of the circadian clock that is involved only in the photic limb of responses.

Whereas much is known about photic signal transduction in the vertebrate circadian system (Reppert and Weaver, 2001), the time-specific aspects of phase shifting are currently not well understood. In the present study, we show that CalB gates photic entrainment of circadian pacemaker. Cytosolic CalB, like other calcium binding proteins, has many functions (Baimbridge et al., 1992), including intracellular $\mathrm{Ca}^{2+}$ buffer (Baimbridge et al., 1982; Mattson et al., 1991), enzyme activator (Morgan et al., 1986; Reisner et al., 1992), and ion channel modulator (Kohr et al., 1991). Nuclear localization of CalB has been reported previously (German et al., 1997); however, the nuclear function of CalB is unknown. Some members of the EF-hand family of $\mathrm{Ca}^{2+}$ binding proteins act as transcriptional repressors, and their affinity for DNA is dependent on $\mathrm{Ca}^{2+}$ (Costigan and Woolf, 2002). CalB in SCN neuronal nuclei may influence the activity of DNA binding proteins by modulating nuclear $\mathrm{Ca}^{2+}$ concentration. $\mathrm{Al}$ ternatively, CalB may act through some as yet undetermined manner. The mechanism whereby reduced cytoplasmic CalB at night blocks the response to light is enigmatic. Nevertheless, our results indicate that $\mathrm{CalB}$ has distinct roles in the cytoplasm and nucleus. Because others have reported that spatially distinct pools 


\section{Treatments during subjective day}

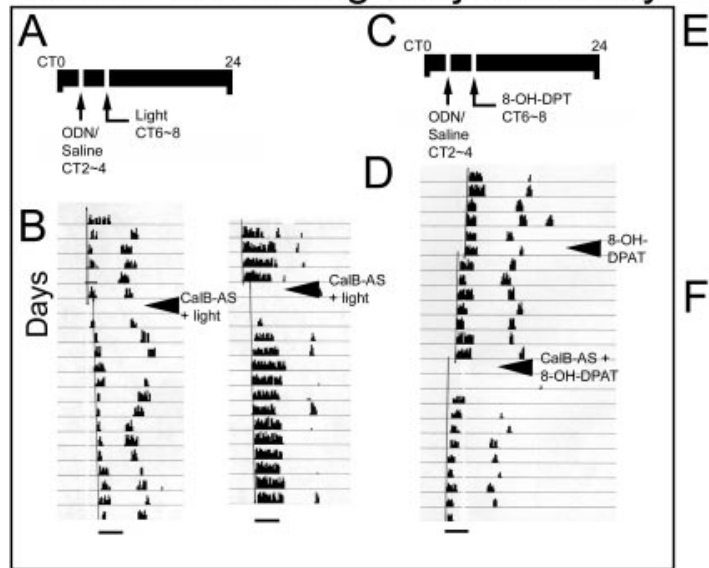

G

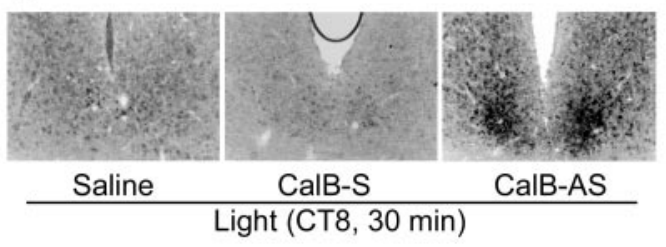

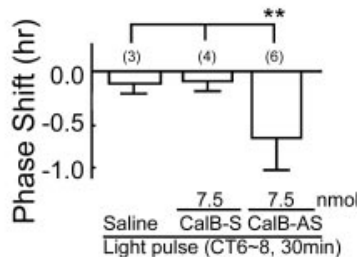

$\mathrm{F}$

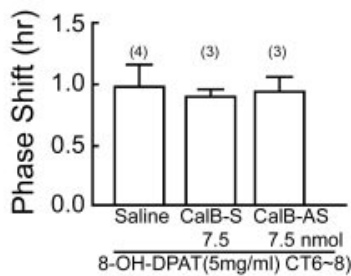

$\mathrm{H}$

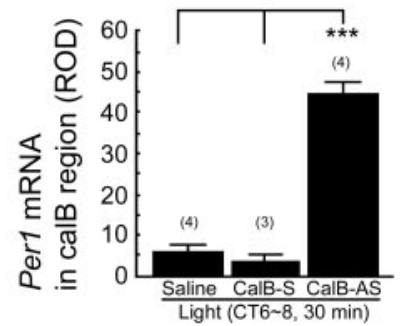

Figure 4. The role of CalB during the subjective day. A, C, Schematic depicts the experimental schedule for light pulse administration during subjective day. CT from 0 to $24 \mathrm{hr}$ is indicated as a line above the actogram. Oligodeoxynucleotides and saline were injected into the third ventricle close to the SCN at CT2-CT4, and a $30 \mathrm{~min}$ light pulse or 8-OH DPAT injection $(5 \mathrm{mg} / \mathrm{kg})$ were given at CT6-CT8. B, Sample actograms from animals given CalB-AS pretreatment before a subjective daytime light pulse. Scale bars: 3 hr. D, Representative actogram from an animals that initially received 8-OH-DPAT alone and subsequently received CalB-AS pretreatment, followed by 8-OH-DPAT. E, Effect of CaIB-AS on locomotor activity rhythm after light pulse. $F$, Effect of CalB-AS on locomotor activity rhythm after 8-OH DPAT treatment. G, Photomicrographs of light-induced expression of Per1 mRNA in the presence of $7.5 \mathrm{nmol}$ of CalB-AS and control injections (saline, CalB-S, $7.5 \mathrm{nmol}$ ) during subjective day in representative animals. $H$, Quantitative analysis of Per1 expression after a subjective daytime light pulse in each treatment condition. Histograms depict data as mean $\pm \mathrm{SEM}$; parentheses give the number of animals per group. ${ }^{* *} p<0.01$; $^{* * *} p<0.001$.

of $\mathrm{Ca}^{2+}$ can influence gene expression in unique ways, the regulation of $\mathrm{Ca}^{2+}$ levels by CalB in specific subcellular compartments may also have different effects on gene expression.

In the circadian system, light-induced phase shifts are dependent on extracellular $\mathrm{Ca}^{2+}$ influx, mediated by the NMDA receptor (Inouye and Shibata, 1994), and intracellular $\mathrm{Ca}^{2+}$ is regulated by two intracellular $\mathrm{Ca}^{2+}$ channels: the inositol trisphosphate receptor (Hamada et al., 1999) and the ryanodine receptor (Ding et al., 1998). Cellular depolarization is accompanied by rapid, transient increases in both cytoplasmic and nuclear calcium concentrations (Sheng et al., 1990). Our results also suggest that nuclear CalB influences calcium-regulated gene expression. During the day, nuclear CalB in SCN neurons likely serves to suppress light-induced Per1 mRNA expression by its $\mathrm{Ca}^{2+}$ buffering effect. The present results also show that light-induced Per1 mRNA expression and phase shifts of locomotor activity rhythm both require light stimulation and a decrease in CalB protein content in the nucleus.

In summary, the present studies indicate that the subcellular localization of CalB is under circadian control. Not only is the temporal gating of photic sensitivity of the circadian system related to the intracellular localization of CalB protein within a subset of SCN neurons, but also the location CalB protein itself may be part of the physiological mechanism responsible for this gate.

\section{References}

Aida R, Moriya T, Araki M, Akiyama M, Wada K, Wada E, Shibata S (2002) Gastrin-releasing peptide mediates photic entrainable signals to dorsal subsets of suprachiasmatic nucleus via induction of Period gene in mice. Mol Pharmacol 61:26-34.

Akiyama M, MinamiY, Nakajima T, Moriya T, Shibata S (1999) Inhibition of light- or glutamate-induced mPerl expression represses the phase shifts into the mouse circadian locomotor and suprachiasmatic firing rhythms. J Neurosci 19:1115-1121.

Albrecht U, Sun ZS, Eichele G, Lee CC (1997) A differential response of two putative mamalian circadian regulators, mper1 and mper2, to light. Cell 91:1055-1064.

Baimbridge KG, Miller JJ, Parkes CO (1982) Calcium binding protein distribution in rat brain. Brain Res 239:519-525.

Baimbridge KG, Celio MR, Rodgers JH (1992) Calcium binding proteins in the nervous system. Trends Neurosci 15:303-308.

Branch AD (1998) A good antisense molecule is hard to find. Trends Biochem Sci 23:45-50.

Bryant DN, LeSauter J, Silver R, Romero M-T (2000) Retinal innervation of calbindinD28K cells in the hamster suprachiasmatic nucleus: ultrastructural characterization. J Biol Rhythms 15:103-111.

Costigan M, Woolf CJ (2002) No DREAM, no pain. Closing the spinal gate. Cell 108:297-300.

Ding JM, Buchanan GF, Tischkau SA, Chen D, Kuriashkina L, Faiman LE, Alster JM, McPherson PS, Campbell KP, Gillette MU (1998) A neuronal ryanodine receptor mediates lightinduced phase delays of the circadian clock. Nature 394:381-384.

Estibeiro P, Godfray J (2001) Antisense as a neuroscience tool and therapeutic agent. Trends Neurosci 11:S56-S62.

German DC, Ng MC, Liang CL, McMahon A, Iacopino AM (1997) Calbindin-D28k in nerve cell nuclei. Neuroscience 81:735-743.

Hamada T, Liou SY, Fukushima T, Maruyama T, Watanabe S, Mikoshiba K, Ishida N (1999) The role of inositol trisphosphate-induced $\mathrm{Ca}^{2+}$ release from IP3-receptor in the rat suprachiasmatic nucleus on circadian entrainment mechanism. Neurosci Lett 263:125-128.

Hamada T, LeSauter J, Venuti JM, Silver R (2001) Expression of Period genes; rhythmic and non-rhythmic compartments of the suprachiasmatic nucleus pacemaker. J Neurosci 21:7742-7750.

Inouye ST, Shibata S (1994) Neurochemical organization of circadian rhythm in the suprachiasmatic nucleus. Neurosci Res 20:109-130.

Jobst EE, Allen CN (2002) Calbindin neurons in the hamster suprachiasmatic nucleus do not exhibit a circadian variation in spontaneous firing rate. Eur J Neurosci 16:2469-2474.

King DP, Takahashi JS (2000) Molecular genetics of circadian rhythms in mammals. Annu Rev Neurosci 23:713-742.

Klein DC, Moore RY, Reppert SM, eds (1991) Suprachiasmatic nucleus: the mind's clock. New York: Oxford UP.

Kohr G, Lambert CE, Mody I (1991) Calbindin-D28K (CaBP) levels and calcium currents in acutely dissociated epileptic neurons. Exp Brain Res 85:543-551.

LeSauter J, Kriegsfeld LJ, Hon J, Silver R (2002) Calbindin-D(28K) cells selectively contact intra-SCN neurons. Neuroscience 111:575-585.

Mattson MP, Rychlik B, Chu C, Christakos S (1991) Evidence for calciumreducing and excito-protective roles for the calcium-binding protein calbindin-D28K in cultured hippocampal neurons. Neuron 6:41-51.

Moore RY (1996) Entrainment pathways and the functional organization of the circadian system. Prog Brain Res 111:103-119. 
Morgan DW, Welton AF, Heick AE, Christakos S (1986) Specific in vitro activation of $\mathrm{Ca}, \mathrm{Mg}$-ATPase by vitamin $\mathrm{D}$-dependent rat renal calcium binding protein (calbindin D28K). Biochem Biophys Res Commun 138:547-553.

Nicot A, Pfaff DW (1997) Antisense oligodeoxynucleotides as specific tools for studying neuroendocrine and behavioral functions: some prospects and problems. J Neurosci Methods 71:45-53.

Ogawa S, Pfaff DW (1998) Current status of antisense DNA methods in behavioral studies. Chem Senses 23:249-255.

Ogawa S, Brown HE, Okano HJ, Pfaff DW (1995) Cellular uptake of intracerebrally administered oligodeoxynucleotides in mouse brain. Regul Pept 59:143-149.

Reisner PD, Christakos S, Vanaman TC (1992) In vitro enzyme activation with calbindin-D28k, the vitamin D-dependent $28 \mathrm{kDa}$ calcium binding protein. FEBS Lett 297:127-131.

Reppert SM, Weaver DR (2001) Molecular analysis of mammalian circadian rhythms. Annu Rev Physiol 63:647-676.
Shearman LP, Zylka MJ Weaver DR, Kolakowski Jr LF, Reppert SM (1997) Two period homologs: circadian expression and photic regulation in the suprachiasmatic nuclei. Neuron 19:1261-1269.

Sheng M, McFadden G, Greenberg ME (1990) Membrane depolarization and calcium induce $\mathrm{c}$-fos transcription via phosphorylation of transcription factor CREB. Neuron 4:571-582.

Shigeyoshi Y, Taguchi K, Yamamoto S, Takekida S, Yan L, Tei H, Moriya T, Shibata S, Loros JJ, Dunlap JC, Okamura H (1997) Light-induced resetting of a mammalian circadian clock is associated with rapid induction of the mPer1 transcript. Cell 91:1043-1053.

Silver R, Romero MT, Besmer HR, Leak R, Nunez JM, LeSauter J (1996) Calbindin-D28K cells in the hamster SCN express light-induced Fos. NeuroReport 7:1224-1228.

Tominaga K, Shibata S, Ueki S, Watanabe S (1992) Effects of 5-HT1A receptor agonists on the circadian rhythm of wheel-running activity in hamsters. Eur J Pharmacol 214:79-84. 